SCIENTIFIC LETTER

\title{
Lower 24 hour urinary sodium concentrations are associated with more severe symptoms in subjects with vasovagal syncope
}

\author{
J L Hampton, S W Parry, R A Kenny, J L Newton
}

Heart 2004;90:687-688. doi: 10.1136/hrt.2003.020115

$\mathrm{V}$ sovagal syncope (VVS) is a common cause of syncope characterised by hypotension, with or without bradycardia, which results in cerebral hypoperfusion and resultant collapse with loss of consciousness. One of the main triggers for VVS (and the pathophysiological cornerstone of the head up tilt table test) is venous pooling in the lower limbs and relative central hypovolaemia. ${ }^{1}$

Despite uncertainty as to the underlying pathophysiology, stratagems to increase blood volume by increasing fluid and salt intake have been shown to be beneficial in the management of VVS. Salt supplementation improves orthostatic tolerance and increases baroreceptor sensitivity in patients with posturally related syncope. ${ }^{2}$ The main determinant of response to salt loading appears to be a 24 hour urinary sodium excretion of $<170 \mathrm{mmol}^{3}$

The aim of this study was to determine the relation between 24 hour urinary sodium excretion and both symptom severity, and tilt induced haemodynamic changes, in subjects with VVS.

\section{METHODS}

From our database we retrospectively identified 68 consecutive patients who attended our tertiary referral syncope unit with a diagnosis of VVS, defined as positive head up tilt with symptom reproduction and in whom a 24 hour urinary sodium collection had been carried out within six weeks of the tilt.

Subjects were excluded if they were taking medication which could be an attributable cause for their VVS and also if they were taking diuretics or mineralocorticoids at the time of the 24 hour urinary collection.

The haemodynamic data for each subject's head up tilt test was reviewed by two independent assessors blinded to the urinary sodium results and classified by the old (type 1, 2A, 2B, 3) and new (classical (C) and dysautonomic (D)) VASIS classifications, ${ }^{4}$ providing both an indicator of haemodynamic parameters as well as possible dysautonomia. Head up tilts all followed the unit protocol $^{5}$ and were sequentially either 40 minute passive head up tilt at $70^{\circ}$, or $800 \mu \mathrm{g}$ glyceryl trinitrate provoked or isoproterenol tilt.

Serum electrolytes and renal function within three months of the 24 hour urine collection were reviewed. In order to determine the severity and type of symptoms at the time the 24 hour collection was carried out, each subject's highly detailed clinical pathway pro forma was reviewed by an observer who was blinded to the urinary sodium and head up tilt results. Symptoms were arbitrarily classified into severe and disabling (that is, presyncope, syncope occurring more than twice in a month, or unheralded and/or injurious syncope) or minimally disabling (less frequent syncope or presyncope).
Twenty four hour urine samples were collected in a standardised manner and all patients were advised of the protocol verbally and via a written instruction sheet.

Comparisons were made using the Instat statistical package. Differences between groups were by MannWhitney U Test for non-parametric variables. A probability value of $\mathrm{p}<0.05$ was considered significant.

\section{RESULTS}

Sixty eight subjects, of whom 47 were female (69\%), were included in the study. All 68 subjects were diagnosed with VVS, as determined by head up tilt with symptom reproduction, and had completed a 24 hour urine collection for sodium.

Serum electrolytes and renal function were available in 59 $(87 \%)$ subjects. All values were within the normal reference range for our laboratory with mean (SD) values of sodium, potassium, urea and creatinine as follows: 139 (2.5) $\mathrm{mmol} / \mathrm{l}$, 4.3 (0.3) mmol/l, 4.6 (1.1) mmol/l, 89 (13) mmol/l.

When considering symptom severity, those with more severe and disabling symptoms $(\mathrm{n}=38)$ had significantly lower 24 hour urinary sodium concentrations compared to those with less severe symptoms $(\mathrm{n}=30, \mathrm{p}<0.0001)$ (table 1).

There was no significant relation between 24 hour urinary sodium concentration and either VASIS classification I or II (table 1). The VASIS classifications dependent upon symptoms are shown in fig 1 and indicate that the subjects with the more severe symptoms were predominantly of the classical rather than the dysautonomic type.

\section{DISCUSSION}

VVS is a heterogenous condition in which the pathophysiology remains unclear. However, low dietary salt is believed to contribute to the low intravascular volume which precipitates the Bezhold-Jarisch reflex resulting in VVS. Previous studies have shown that salt supplementation improves orthostatic tolerance and baroreflex sensitivity in those with unexplained syncope. ${ }^{2}$ In this study we have shown a clear association between 24 hour urinary sodium concentrations and increasing symptom severity in patients with tilt positive VVS. This further supports the hypothesis that changes in baroreceptor sensitivity may play a role in the pathophysiology of VVS.

These findings could be related to either decreased intake of sodium in sufferers or abnormalities in sodium metabolism or excretion. Increasing salt intake improves symptomatology in those with VVS and points more towards dietary deficiency; however, a genetic abnormality of salt metabolism could also play a role. Recent work from our group has pointed towards the potential for VVS as a complex genetic disease and this study may direct subsequent candidate gene studies. 
Table 1 Characteristics, blood pressure, and 24 hour urinary sodium concentrations in 68 patients with definite VVS

\begin{tabular}{|c|c|c|c|c|c|c|c|c|c|}
\hline & \multirow[b]{2}{*}{ Total } & \multicolumn{2}{|l|}{ Symptoms } & \multicolumn{4}{|c|}{ VASIS I classification } & \multicolumn{2}{|c|}{ VASIS II classification } \\
\hline & & Severe & Mild & 1 & $2 A$ & 2B & 3 & C & D \\
\hline $\mathrm{n}$ & 68 & 38 & 30 & 31 & 5 & 6 & 26 & 53 & 15 \\
\hline Age (years) & $43(17)$ & $42(17)$ & $46(16)$ & $46(17)$ & $46(11)$ & $50(18)$ & $39(16)$ & $44(16)$ & $42(18)$ \\
\hline $\begin{array}{l}\text { Systolic blood pressure } \\
\text { (mm Hg) }\end{array}$ & $126(19)$ & $127(19)$ & $126(22)$ & $125(23)$ & $124(15)$ & $126(21)$ & $129(18)$ & $127(21)$ & $126(16)$ \\
\hline Diastolic blood pressure & & 2द्रा & $66(12)$ & 年 & $64114)$ & $64(15)$ & $63(14)$ & (2) & \\
\hline $\begin{array}{l}(\mathrm{mm} \mathrm{Hg}) \\
24 \text { hour urinary sodium }\end{array}$ & $64(12)$ & $63(11)$ & $66(12)$ & $63(10)$ & $64(14)$ & $64(15)$ & $63(14)$ & $64(12)$ & $64(10)$ \\
\hline (mmol/24 hours) & $162(65)$ & $121(35)^{*}$ & $213(57)^{*}$ & $163(69)$ & $226(84)$ & $124(42)$ & $158(55)$ & $160(67)$ & $169(58)$ \\
\hline
\end{tabular}

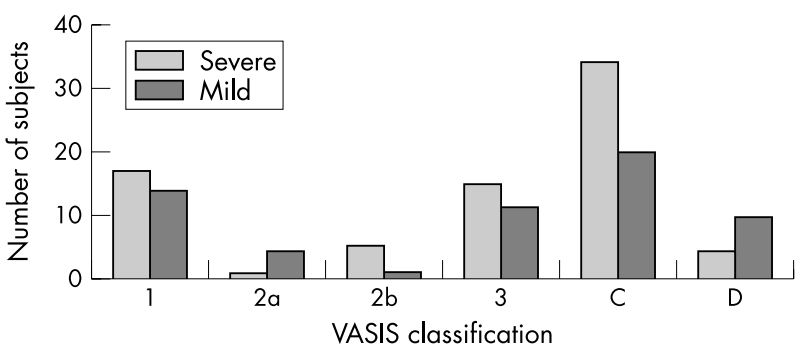

Figure 1 Number of subjects in each VASIS class with severe compared to mild symptoms (VASIS I classification: 1, 2A, 2B, 3; VASIS II classification: classical (C), dysautonomic (D) 4 .

This study does have limitations as it is retrospective and examines a selected population. There was no relation between urinary salt concentrations and haemodynamic parameters during tilt, but this may be related to the small numbers of subjects in some VASIS classes. Furthermore, the relation between urinary sodium output and severity of symptoms is associative and not necessarily causative. It does raise the question of how VVS, with its characteristic sporadic symptoms, could be triggered by sodium depletion. Despite this, and in keeping with previous studies, we would suggest that 24 hour urinary sodium concentration is a useful indicator in those with VVS. It may allow those with salt deficiencies to be identified and may also predict which patients are likely to develop more disabling symptoms. It might also indicate those patients who would benefit most from increased salt intake, a benign and effective intervention for many sufferers of VVS.

\section{Authors' affiliations}

J L Hampton, S W Parry, R A Kenny, J L Newton, Cardiovascular Investigation Unit, University of Newcastle upon Tyne, Care of the Elderly Offices, Royal Victoria Infirmary, Newcastle, UK

Correspondence to: Dr Julia L Newton, Cardiovascular Investigation Unit, University of Newcastle upon Tyne, Care of the Elderly Offices, Royal Victoria Infirmary, Newcastle, UK, NE1 4LP;

julianewton@blueyonder.co.uk

Accepted 25 September 2003

\section{REFERENCES}

1 Parry SW, Kenny RA. Tilt table testing in the diagnosis of unexplained syncope. QJM 1999:92:623-9.

2 Cooper VL, Hainsworth R. Effects of dietary salt on baroreceptor tolerance, blood pressure and baroreceptor sensitivity in patients with syncope. Clin Auton Res 2002;4:236-41.

3 El-Sayed H, Hainsworth R. Salt supplement increases plasma volume and orthostatic tolerance in patients with unexplained syncope. Heart 1996;75: 134-40.

4 Brignole M, Menozzi C, Del Rosso A, et al. New classification of haemodynamics of vasovagal syncope: beyond the VASIS classification. Europace 2000;2:66-76.

5 Kenny RA. O'Shea D. Parry SW. The Newcastle protocols for head-up tilt table testing in the diagnosis of vasovagal syncope, carotid sinus hypersensitivity, and related disorders. Heart 2000;83:564-9. 